\title{
A ToF-MS with a Highly Efficient Electrostatic Ion Guide for Characterization of Ionic Liquid Electrospray Sources
}

\author{
Subha Chakraborty, Caglar Ataman, Daniel G. Courtney, Simon Dandavino, \\ Herbert Shea
}

Microsystems for Space Technologies Laboratory, EPFL, Neuchatel, Switzerland

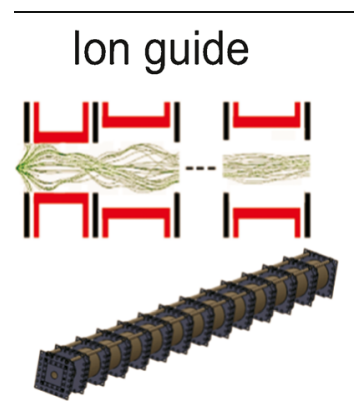

\begin{abstract}
We report on the development of a time-of-flight (ToF) mass spectrometer with a highly efficient electrostatic ion guide for enhancing detectability in ToF mass spectrometry. This $65-\mathrm{cm}$ long ion guide consists of 13 cascaded stages of Einzel lens to collect a large fraction of emitted charges over a wide emission angle and energy spread for time-of-flight measurements. Simulations show that the ion guide can collect $100 \%$ of the charges with up to $23^{\circ}$ emission half-angle or $30 \%$ energy spread irrespective of their specific charge. We demonstrate this ion guide as applied to electrospray ion sources. Experiments performed with tungsten needle electrospraying the ionic liquid EMI$\mathrm{BF}_{4}$ showed that up to $80 \%$ of the emitted charges could be collected at the end of
\end{abstract} the flight tube. Flight times of monomers and dimers emitted from the needles were measured in both positive and negative emission polarities. The setup was also used to characterize the electrospray from microfabricated silicon capillary emitters and nearly $30 \%$ charges could be collected even from a 40 emission half-angle. This setup can thus increase the fraction of charge collection for ToF measurement and spray characteristics can be obtained from a very large fraction of the emission in real time.

Key words: Mass spectrometry, Electrostatic lens, Ion guide

Received: 29 November 2013/Revised: 9 April 2014/Accepted: 9 April 2014/Published online: 16 May 2014

\section{Introduction}

$\mathrm{T}$ ime-of-flight (ToF) mass spectrometers are analytical instruments capable of measuring a wide spectrum of charge $(q)$ to mass $(m)$ ratios in real-time [1]. First developed in 1948 by Cameron and Egger [2]ToF mass spectrometers have evolved to achieve higher mass resolution and sensitivity through developments such as time-lag focusing techniques [3], plasma and laser desorption techniques [1, 4, 5], and tandem ToF-ToF [6]. The introduction of electrospray ionization (ESI) techniques [7-9] has significantly enhanced the capabilities of ToF-MS instruments, especially for analyzing biological macromolecules.

One of the key parameters that define efficiency of any mass spectrometry (MS) system is the transmission of emitted charges to the detector and thereby detectability [10]. In contrast to MS systems that sample spectral

Electronic supplementary material The online version of this article (doi:10.1007/s13361-014-0914-z) contains supplementary material, which is available to authorized users.

Correspondence to: Subha Chakraborty; e-mail: subha.chakraborty@epfl.ch intensities serially, ToF can yield a complete spectrum of all detected particles with each measurement, thereby enabling analysis of dynamic systems. When combined with means to maximize the measured proportion of emitted particles [11-16], the completeness of each spectral measurement is improved through enhanced detectability attributable to increased signal and/or greater accommodation of anisotropic spatial spreading. Numerous applications may exploit this trait. These include uses of electrospray sources, the focus of this paper, and introduced below, and chemical analyses, such as high speed chromatography, pyrolysis, desorption, on-line analysis of gas mixtures, etc. [17, 18]. Here, fast attainment of the entire mass spectrum is important because of rapidly changing sample composition. For example, investigation of kinetics and mechanisms of thermal decomposition and production of intermediate radicals in pyrolysis poses an essential criterion of real-time monitoring of the mass-spectrum [19, 20]. Monitoring time evolution of growing bacteria [21, 22] and their temporal characteristics, especially for bioterror agents, require mass spectra within minutes [23] for clinical and environmental applications. For high-speed ion-imaging applications, an entire mass spectrum is required from each pixel, and 
imaging an entire object requires very fast acquirement of the entire spectra [24].

Apart from ionization technique in ToF-MS, electrospray has gained significant importance in the field of spacecraft propulsion $[25,26]$ and focused ion beam applications [27, 28]. In the cited applications, and in particular when spraying ionic liquids (ILs), ES source often are operated close to a transition region between emission of droplets and direct field evaporation of much less massive ions with significant variations on performance due to each species. Also, over the life of the source, the spray properties from IL sources can vary over time due to electrochemical reactions [29] or due to instantaneous formation and annihilation of Taylor cones $[30,31]$. By providing a real-time capture of all particles detected over a wide range of species mass comprising individual ions and droplets, ToF-MS is therefore a logical and ideal method for analyzing ES designed for propulsion and focused ion beam (FIB) applications. Depending on the emitter type, the emission from such IL source can take place with a half-angle up to $40^{\circ}$ [32]. Furthermore, evidence exists for localized angular zones of ion and droplet emission [11, 33, 34] with higher $q / m$ components usually away from the axis of emission. Beam energies are also known to spread by up to $10 \%$ about nominal with additional secondary energy peaks spread over a much wider range $[35,36]$. These features pose an additional requirement of capturing entire mass spectra from a wide emission angle and energy range on the ToF-MS for characterizing such IL sources. To accommodate a wide angle and energy range over a $10-100-\mathrm{cm}$ long flight tube is a challenge, particularly when the emitted current is low (nA- $\mu \mathrm{A}$ range). This research seeks to improve detectability of ToF-MS for these applications by enhancing the accommodation for angular spreading and energy distributions at each measurement.

ToF measurements on IL ES sources often are performed only on the center portion of the beam [11, 27]. Angleresolved ToF spectrometry, in which the relative angle between the emitter and the detector [37] or the distance between the emitter and the detector is changed [38], has also been reported; however, capturing a wider fraction of emission is beneficial because this gives real time mass spectra over the entire emission cross-section at once. One way to collect a large fraction of the emission is using a large detector [34, 38, 39]; alternatively, Einzel lenses focus charges over a certain angle and energy range on the detector by electrostatic deflection [12-16], which allows for a reduced compromise between sizing [21] and detectability [10].

In addition to increasing ion transmission to the detector for ToF measurement, Einzel lenses are also used in ILbased FIB techniques for increasing spot intensity after allowing ions through a narrow aperture [27, 28]. The required focal length of a single-stage three-electrode lens can be achieved by using mechanically optimized dimensions and shapes of the electrodes and/or by varying the focusing potential [40]. However, for diagnostics over wide and arbitrary emission angle and energy spread, as in the case of IL sources, aberrations [12, 40] (both spherical and chromatic aberration) are a problem, resulting in significant reduction in detected signal. Aberration can be reduced by using axially asymmetric three-electrode lenses [12, 40, 41], however, achieving minimum spherical aberration in tandem with minimum chromatic aberration is difficult. In other words, if mono-energetic charges at different angles are focused on a detector, charges with different energies emerging at the same angle are defocused. Use of singlestage asymmetric Einzel lenses in MS applications has been reported in literature $[12,16]$. However, optimum performance of a single lens is achieved at low focal lengths, most suitable for field emission guns [41, 42]. The different lens geometries reported in [41] achieve minimum spherical aberration at focal lengths much shorter than that required in ToF measurements, and increasing the focal length drastically increases aberration. For example, in [12], the asymmetric Einzel lens could only focus up to an emission half-angle of $13^{\circ}$ and only up to $13 \%$ of the emission current from pulled silica capillary electrospray emitters was available for ToF measurement.

In this paper, we report on development of a ToF-MS with an electrostatic ion guide that can collect all charges within nearly $23^{\circ}$ emission half-angle and with broad energy spread from ionic liquid electrospray emitters within a particular range of lens voltage. This ion guide is comprised of 13 stages of three-element symmetric lens all along the length of the flight tube to guide the charges to a Faraday cup detector of $1-\mathrm{cm}$ diameter placed at $65-\mathrm{cm}$ distance from the source for ToF measurements. The idea of the ion guide stems from the use of multi-stage lenses in different configurations to guide ions through the drift tube [40,43]. In principle, this ion guide compensates for over-focusing or under-focusing in every stage for different energies and angles and thus increases the fraction of charges collected, similar to the ion guide reported in $[44,45]$ for an ion mobility mass spectrometry (MS). Each stage focuses the charges within the length of the next stage and, thus, along the tube length, keeps the charges close to the axis of the tube. Using an electron amplifier [12] instead of a Faraday cup can increase the detected signal significantly, but since the purpose of this work is to increase detectability using the ion guide, a Faraday cup has been utilized to capture the real detected current for ToF measurements.

Due to deceleration and acceleration at each stage, the ion guide increases the flight time of the charges, which is equivalent to having a longer flight tube without compromising on detectability. However, this effect does present a limitation of the device because of non-uniform increases in flight time over emission angle. Flight time non-uniformity corresponds to a reduction in $q / m$ sensitivity and, hence, the benefits of enhanced detectability and longer flight times must be traded against this limitation. This characteristic is explored through simulations and experiments below. 
The performance of our setup was first characterized with electrochemically etched tungsten needles, which provide nearly mono-energetic emission in pure ionic mode with the

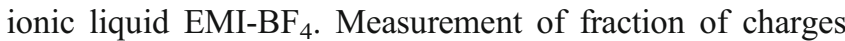
collected and ToF were performed in positive and negative polarities of emission from the needles, and the results are consistent with the simulation results. Subsequently, the validated ion guide was applied to characterization of microfabricated capillary emitters operating in the mixed ion/droplet regime $[39,46]$.

\section{ToF-MS System and Ion Guide Design}

The ion guide we report upon here can be dimensioned for any beam energy, $q / m$ ratio, and other constraints such as readout electronics. We show here the reasoning behind our choice of lens dimensions for use with ionic liquid electrospray sources based on externally wetted tungsten needle emitters and on microfabricated capillary emitters. The needles have been previously reported to emit 100-200 nA nearly mono-energetic pure ions within

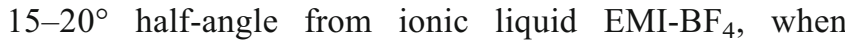
operated at emission voltage $V_{e m} \sim 1.5 \mathrm{kV}$ [47]. The capillary emitters have been previously reported to emit 1-2 $\mu \mathrm{A}$ mixed ion/droplet with nearly $40^{\circ}$ half-angle operating at $V_{e m} \sim 1 \mathrm{kV}$ [32]. The emission from EMI$\mathrm{BF}_{4}$ can contain the charge species as shown in Table 1 depending on the emitter configuration.

The kinetic energy of the emitted charges is given by (ignoring for this initial analysis the energy spread and emission energy losses)

$\frac{1}{2} m v^{2}=q V_{e m}$

where $v$ is the speed of the charge. For a flight length $L$, the flight time is

$T o F=L\left(\frac{m}{2 q V_{e m}}\right)^{1 / 2}$

If no focusing system is used, there is a trade-off between: (1) the fraction of emitted beam that can be collected with a small Faraday cup detector, and (2) the ToF, which must be at least a few $\mu$ s for accurate detection by fast amplifiers. If the detector is placed close to the emitter, it can collect a significant fraction of the emitted charges, but the flight time is very low, requiring very high bandwidth of the current detection circuit. On the other hand, placing the detector far away would increase the flight time; however, the low collected currents on the Faraday cup detector would lead to an unacceptably small signal-to-noise ratio, even when state-of-the-art high bandwidth trans-impedance amplifiers are used.
Dimensions of the ToF MS Flight Tube The minimum flight time (i.e., minimum tube length) is in part set by the speed of the trans-impedance amplifier at the output of the Faraday cup. We take into consideration the trans-impedance gain of a commercial state-of-the-art FEMTO DHPCA100 current amplifier [48]. A gain setting of at least $10^{6} \mathrm{~V} / \mathrm{A}$ would be required to convert $100 \mathrm{nA}$ current into a measurable $100 \mathrm{mV}$ output. This would lead to a bandwidth of $1.8 \mathrm{MHz}$ [48] of the current amplifier. Therefore, the flight time of the fastest ions should be no less than $10 \mu \mathrm{s}$. As discussed, we use a maximum voltage $\pm 2 \mathrm{kV}$ and the corresponding flight time of the fastest ions $\mathrm{BF}_{4}{ }^{-}$would be $10 \mu$ s for a flight tube length $L \sim 65-\mathrm{cm}$. For other emitted species in Table 1, the flight time would be longer. Our ion guide is made with commercially (Kimball Physics [49]) available square plates of size $5 \mathrm{~cm}(7 \mathrm{~cm}$ diagonal). On the basis of these criteria, a $65-\mathrm{cm}$ long flight tube is chosen, which has a $7-\mathrm{cm}$ inner diameter.

Ion Guide Design and Performance Simulation In this section, we provide the simulated performance of the ion guide in terms of its charge collection capabilities and ToF for emission having angular and energy spread. Since each stage of the ion guide focuses the charges within the length of the next stage, this gives us the flexibility of using symmetric lens stages with commercially available dimensions [49] for modularity and speed of assembly and yet giving high transmission over wide emission half-angle and energy. The central electrode of each stage is a hollow cylinder at the focusing voltage $V_{\text {lens }}$ and the other two are grounded electrodes made of square plates with circular openings. Simulations of ion trajectory have been performed in SIMION ion simulator (ver. 8.0) with different values of $V_{\text {lens }}$ and $V_{e m}$, for the available lengths $l$ and diameters $d$ of the cylinders [49] and the openings of the grounded plates. The distance between the electrodes is taken as 3-mm, considering the practical aspects of alignment and wiring. The emitter, considered as a point source, is 6-mm in front of the first ground electrode so that a gate electrode can later be inserted in between for ToF measurements. For different lens geometries, the focusing capabilities are independent of the $q / m$ ratio of the charges and depend only on the ratio $V_{\text {lens }} / V_{e m}$. For different single-stage symmetric Einzel lens configurations, as shown in Figure 1a, the maximum

Table 1. Types of Charges Emitted from EMI-BF 4

\begin{tabular}{|c|c|c|}
\hline Polarity of emission & Type of charge & $q / m$ \\
\hline \multirow[t]{4}{*}{ Positive } & Monomer $\mathrm{EMI}^{+}$ & $0.009 e / \mathrm{u}$ \\
\hline & Dimer $\left(\mathrm{EMI}^{-\mathrm{BF}_{4}}{ }^{-}\right) \mathrm{EMI}^{+}$ & $0.0032 e / \mathrm{u}$ \\
\hline & 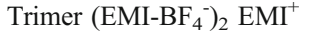 & $0.002 \mathrm{e} / \mathrm{u}$ \\
\hline & Droplets & $10^{-4}$ to $10^{-6} \mathrm{e} / \mathrm{u}$ \\
\hline \multirow[t]{4}{*}{ Negative } & Monomer $\mathrm{BF}_{4}^{-}$ & $-0.011 e / \mathrm{u}$ \\
\hline & 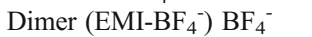 & $-0.0035 e / \mathrm{u}$ \\
\hline & 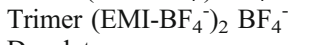 & $-0.002 \mathrm{e} / \mathrm{u}$ \\
\hline & Droplet & $-10^{-4}$ to $-10^{-6} e / \mathrm{u}$ \\
\hline
\end{tabular}


emission half-angle up to which $100 \%$ of the emitted charges can be collected on the $1-\mathrm{cm}$ diameter detector are simulated first assuming uniform charge density over the emission half-angle. Then, with this first-stage, the other stages of the ion guide have been optimized for maximum collection. The final dimensions of the ion guide are given in Table 2. In Figure 2a, the maximum emission half-angle up to which all of the emitted mono-energetic charges can be collected on the detector using the ion guide and the firststage only have been plotted against the ratio $V_{\text {lens }} / V_{e m}$.

As seen from the ion trajectories in Figure $1 \mathrm{~b}$ and from the plots in Figure 2a, the first-stage Einzel lens alone focuses mono-energetic charges within up to $9^{\circ}$ to the collector. In contrast, the ion guide can collect all monoenergetic charges within $\theta_{e m}=23^{\circ}$ half-angle. Also, as $V_{e m}$ is a measure of the kinetic energy of the emitted particles, it can be inferred from the broad maxima in the plot that charges with nearly $30 \%$ spread of initial kinetic energy within $15^{\circ}$ half-angle can also be collected for a given optimum lens voltage. This would mean that for an externally wetted needle emitter, the ion guide can collect all charges. For capillary emitters, which have beam halfangles up to $40^{\circ}, 25 \%$ for mono-energetic charges are collected. For ions confined within $10^{\circ}$, up to $30 \%$ energy spread can be accommodated from the capillary emitters. However, since the beam is actually concentrated near the axis $[47,32]$, not uniformly distributed in the beam crosssection, the energy band and fraction of charge collection can be higher. With an optimized asymmetric single-stage as in [41], the collected fraction of the first-stage and the ion guide is expected to be even higher.

Increase in Time of Flight in the Ion Guide The ion guide leads to an increase in the flight time of the charges compared to the value obtained from (2). In a single-stage lens, the change in average speed of the particles is typically negligible since the deceleration and acceleration take place only within the length of the lens, which is much smaller than the total length of the flight tube. This is not the case for the long ion guide. In every stage of the ion guide, the charges are decelerated and then accelerated back to nearly their initial speed and as a result the average axial speed of the charges is lower than the initial speed. In order to quantify the change in flight time due to the ion guide, a ToF correction factor, which depends on the lens voltage, is defined in Equation 3 as:

ToF correction factor $=\frac{T o F\left(V_{\text {lens }}\right)}{T o F\left(V_{\text {lens }}=0\right)}=\frac{T o F\left(V_{\text {lens }}\right)}{L\left(\frac{m}{2 q V_{\text {em }}}\right)^{1 / 2}}$

This allows the correct $q / m$ to be evaluated from the measured ToF. Simulations performed at different combinations of $V_{e m}$ and $V_{\text {lens }}$ show that this ToF correction factor is a function of the ratio $V_{\text {lens }} / V_{e m}$ as plotted in Figure 2b. This factor is independent of the $q / m$ of the charges and the length of the flight tube as long as their kinetic energy is $q V_{e m}$. The ToF of the charges along the axis increases from the value given by (2) by a factor of nearly three as the $V_{\text {lens }} / V_{e m}$ ratio increases from 0 to 1 . This implies that the effective length of the flight tube is increased by the same factor without actually increasing the size of the flight tube.

As shown in Figure 2b, the differences in ToF correction factor at different angles leads to a longer flight time for charges emitted at higher angle than those along the axis. For emission of monomers (e.g., $\mathrm{BF}_{4}^{-}, 87 \mathrm{u},-1 e$ ) from the needles within $15^{\circ}$, the maximum spread in ToF between onaxis and off-axis particles is within $20 \%$ for $V_{\text {lens }} / V_{\text {em }}$ up to

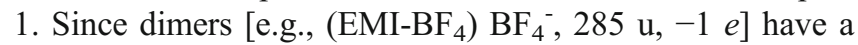
ToF $80 \%$ longer than that of the monomer, the two ion species can easily be identified. As discussed in the Introduction, this spread in flight time and, therefore, a reduction in $q / m$ resolution must be traded against the improved detectability achieved through capturing a greater fraction of the beam current. The ion guide could be employed in tandem with a variable iris near the source to enable selection between an emphasis on detectability, through admitting a large beam angle, or resolution, when restricting transmission to near-axis charges.

\section{Experimental Setup}

The ToF-MS setup consists of (1) a charged particle source (in our case an electrospray emitter assembly), (2) the electrostatic gate, (3) the ion guide, (4) a Faraday cup in a vacuum chamber with a $65-\mathrm{cm}$ long flight tube, and (5) drive electronics.

The Needle Ion Emitter and Its Assembly The needle emitters are fabricated from $3-\mathrm{cm}$ long and $0.5-\mathrm{mm}$ diameter tungsten wires using an electrochemical process as described in $[47,50]$ (see Supplementary Figure S1 for SEM image). The needle is mounted on a $6-\mathrm{mm}$ thick polyoxymethylene (POM) plate, cut to $5-\mathrm{cm}$ side square shape with $0.5-\mathrm{mm}$ diameter hole at the center to mount the needle. A stainless steel plate with $1-\mathrm{mm}$ diameter circular hole at the center is used as the extractor electrode and the needle is aligned to the extractor aperture using an optical microscope. A tungsten wire loop is created nearly $5-\mathrm{mm}$ beneath the tip of the needle so that a reservoir drop of ionic liquid can be sustained there due to capillarity and can externally wet the sharp tip of the needle with the ionic liquid.

The Ion-Guide and the ToF Measurement Chamber The experimental setup consists of a $15-\mathrm{cm}$ cubic vacuum chamber with a $65-\mathrm{cm}$ long, $7-\mathrm{cm}$ diameter flight tube as shown in Figure 3a. The ion guide, as shown in Figure 3b, slides through the tube and is aligned with the axis of the tube with the grounded square plates in the ion guide 

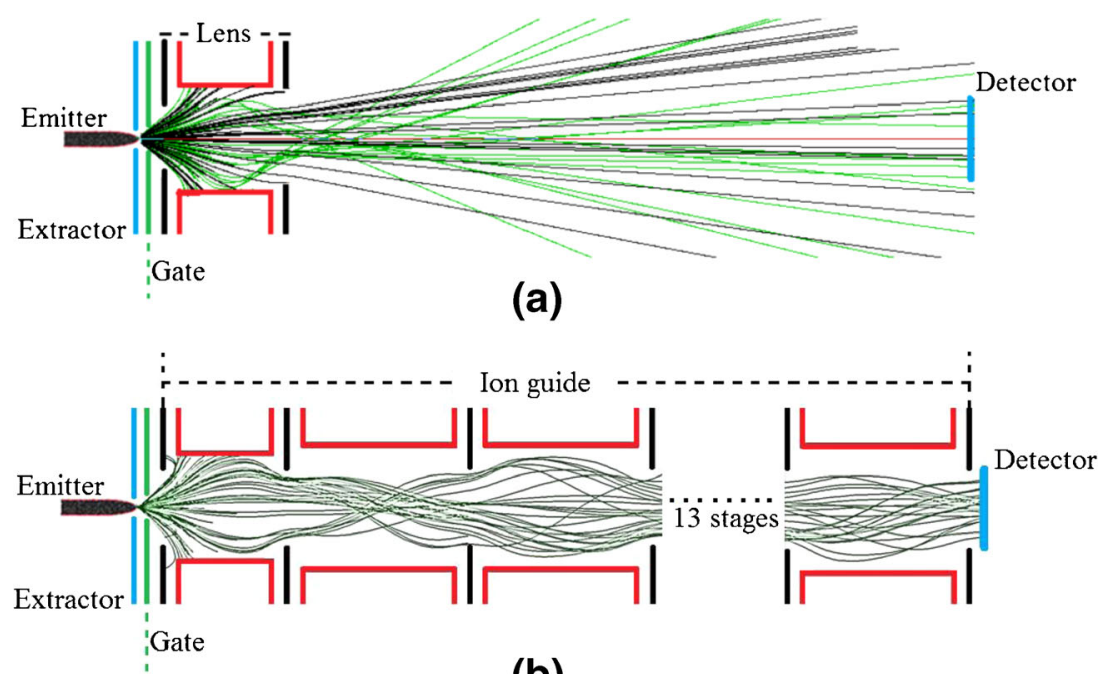

(b)

Figure 1. Schematic of a ToF setup with (a) a single-stage lens, and (b) with the ion guide. In (b), high voltage cylindrical electrodes ( $\mathrm{red}$ ) and grounded plate electrodes (black) are cascaded all along the length of the flight tube to guide the charges to the detector. The simulated ion-trajectories for different initial kinetic energy show the benefit of using the ion guide over a single-stage lens

assembly. The edges of the square metallic plates holding the high voltage $\left(V_{\text {lens }}\right)$ cylindrical electrodes are cut to a round shape in order to avoid electrical contact with the body of the tube. On the side of the ion guide that goes inside the cubic vacuum chamber, the emitter assembly is attached and aligned to it using alumina tubes. A gate electrode, made of a $5-\mathrm{cm}$ square stainless steel plate and a circular central opening with a metallic grid with $85 \%$ optical transparency, is also placed between the emitter and the ion guide, each separated from the other by $3-\mathrm{mm}$. On the other side of the ion guide, a Faraday cup with diameter $10-\mathrm{mm}$ is attached and aligned in the same way. The vacuum chamber is pumped by an Edwards WRG-S pumping station. All experiments are performed with an indicated vacuum pressure less than $10^{-4}$ mbar.

\section{Electronic Setup}

The high voltage positive and negative power supplies for the emitter are implemented using EMCO CA20P and CA20N DC-DC converters [51]. For the ion guide EMCO H30P and H30N DC-DC converters are used [51]. Two AD210 isolation amplifiers [52] with selectable gain settings are used to measure the emitter current directly at the emitter power supplies. For fast switching of the gate signal, a PVX-4140 high-speed pulse generator [53] is used, along with another pair of EMCO Hseries $\pm 3 \mathrm{kV}$ power supplies. The detected current from the Faraday cup is fed to a FEMTO DHPCA100 trans-impedance amplifier in 'low noise' configuration [48] with a gain $10^{6} \mathrm{~V} / \mathrm{A}$ and bandwidth $1.8 \mathrm{MHz}$ with a $50 \Omega$ termination. The control signals for the experiments are generated using a 16-channel analog output system from National Instruments (Ennetbaden, Switzerland). All the signals are recorded using a 16-channel analog input system from National Instruments. The output of the trans-impedance amplifier is monitored using an oscilloscope for ToF measurements and using the analog input channel for measurements of collected fraction of current. All signal generation and monitoring are controlled using Matlab (ver. 7.9).

\section{Experimental ToF and Charge Collection Data with Needle Ion Sources}

To identify the desired operating conditions, we first measure the $\mathrm{I}-\mathrm{V}$ relation of the emitters, recording the emitted current from the needles while sweeping the emitter voltage from $1000 \mathrm{~V}$ to $2000 \mathrm{~V}$ in $5 \mathrm{~V}$ step in both polarities, with no beam focusing and the ToF gate grounded. Emission sets in at around $\pm 1300 \mathrm{~V}$ and the average current increases to nearly $95 \mathrm{nA}$ for positive polarity and $-63 \mathrm{nA}$ for negative polarity as the voltage increases to $\pm 2 \mathrm{kV}$. Stable operation (non-pulsating beam) is observed above \pm 1400 V (see Supplementary Figure S2 for I-V results).

Fraction of Charges Collected with the Ion Guide from the Needles Since stable emission is achieved beyond $\pm 1400 \mathrm{~V}$ in both polarities, experiments have been performed for emitter voltages higher than this value. The emitter voltage $V_{e m}$ is set to different fixed values and the voltage to the ion guide, $V_{\text {lens }}$, is varied from 0 to $1.1 \times V_{e m}$ in 51 steps for each $V_{e m}$. At each step, the emitted and the detected current are recorded for $1 \mathrm{~s}$ with 100 samples/s sampling rate and averaged. In Figure 4a, and b, the fraction of collected charges for three different emitter voltages in both polarities are shown as a function of the $V_{\text {lens }} / V_{\text {em }}$ ratio. The data have been corrected to account for the $85 \%$ optical transmission fraction of the ToF gate installed during the measurements. Simulations of the fraction of 
Table 2. Dimensions of the Ion Guide

\begin{tabular}{llllll}
\hline Element & $\begin{array}{l}\text { Length of deflecting } \\
\text { cylinder } l\end{array}$ & $\begin{array}{l}\text { Diameter of deflecting } \\
\text { cylinder } d\end{array}$ & $\begin{array}{l}\text { Diameter of opening } \\
\text { in the ground electrodes }\end{array}$ & $\begin{array}{l}\text { Spacing between } \\
\text { electrodes }\end{array}$ & $\begin{array}{l}\text { Voltage applied to the } \\
\text { deflecting cylinder }\end{array}$ \\
\hline $\begin{array}{l}\text { First stage } \\
\mathrm{n}^{\text {th }}(n=2 \text { to } 13) \text { stage }\end{array}$ & $\begin{array}{l}25-\mathrm{mm} \\
37.5-\mathrm{mm}\end{array}$ & $\begin{array}{l}25-\mathrm{mm} \\
37.5-\mathrm{mm}\end{array}$ & $25-\mathrm{mm}$ & $3-\mathrm{mm}$ & $3-\mathrm{mm}$ \\
\hline
\end{tabular}

collected charges for mono-energetic emission (with kinetic energy $=q V_{e m}$ ) has been performed assuming a beam shape reported for needles in [47] and are shown in the same figures.

The measured fraction of detected current reaches $80 \%$ for positive polarity at $V_{e m}=1450 \mathrm{~V}$ when the focusing voltage is set to $V_{\text {lens }}=0.6 \times V_{\text {em }}$. Similar values are measured for negative polarity of emission. The collection fraction reaches a value between $40 \%$ and $80 \%$ with $V_{\text {lens }} / V_{e m}$ set between 0.6 and 0.9 . This implies that if the lens voltage is fixed to a value equal to $0.6 \times V_{e m}$, the ion guide can collect charges with kinetic energy spread of nearly $30 \%$. The experimental plots of collected current follow the simulation results quite well. The maximum collection fraction is in the similar range as obtained in $[50,54]$ using large metallic plate to collect current in a large vacuum chamber, and nearly seven times higher than that obtained in [12] with a single-stage lens within similar angular spread.

The collection fraction, although high, does not reach the simulated value of $100 \%$. It is also observed that the range of lens voltage, for which maximum current is detected, is shifted by nearly $V_{\text {lens }} / V_{e m}=0.15$ from the simulation results and is a little broader. Within the range $V_{\text {lens }} / V_{\text {em }}=0.6$ to 0.9 , the experimentally observed collected fraction reduces as $V_{\text {lens }}$ is increased. One possible explanation for these differences is simply practical imperfections of the entire system, such as misalignment and the electric field due to wiring. The spray divergence in our experiments may also be larger than that assumed in the simulation, especially at emission voltages where the detected current is lower. It is also possible that as in [30], emission occurs at a non-zero angle to the ion guide axis and, therefore, all the emitted charges may not enter the ion guide.

In negative polarity (Figure $4 \mathrm{~b}$ ), the measured fraction increases quite sharply between $V_{\text {lens }} / V_{\text {em }}=0$ and $V_{\text {lens }} / V_{\text {em }}=$ 0.1 , a feature not found in the simulations, which were performed assuming only mono-energetic emission with kinetic energy $q V_{e m}$. However, the emitted ions can fragment during flight (e.g., dimers into monomers), which leads to monomers with kinetic energy lower than the emission kinetic energy of the dimers and, as a result, higher fraction of current can be collected at lower lens voltages. This might be another cause for the spread in the Figure 4 beyond the simulation range.

Time of Flight Data for Ion Guide Systems with Needle Ion Emitters The flight time of the ions was recorded at three different values of $V_{e m}$ in both polarities of operation while varying the lens voltage $V_{\text {lens }}$. The gate signal is triggered at time $t=0$ with a fall-time of $<100 \mathrm{~ns}$. Monomers and dimers are observed in the traces in both polarities and their flight times are recorded. The observed spread is within $20 \%$ at all lens voltages as estimated from the simulated effect of the ion guide accepting wide emission angles. Referring to Figure $2 b$, flight times are expected to increase at wider angles. We have, therefore, selected the minimum flight time, corresponding to the beginning of each species step in the ToF trace, in comparing against the $0^{\circ}$ simulations discussed below.

In Figure 5, the measured flight time of the monomers and dimers are plotted against the lens voltage for fixed emitter voltages. The simulation results based on (2) and ToF correction factor for the corresponding situations for $\theta_{e m}=0^{\circ}$ are also shown on the same figures for comparison. The experimental results are in excellent agreement with the simulations for monomers and dimers of both polarities, showing that the ion guide can be used to greatly increase the collected current, while allowing accurate ToF acquisition (the requirements on the trans-impedance amplifier are greatly relaxed compared with the case of a single Einzel lens since the current values are much higher, thus allowing lower gain).

\section{Experimental Results Using Microfabricated Capillary Emitters}

After characterizing the ToF system and the ion guide for needle emitters, the system was used for spray characterization of capillary-fed internally wetted microfabricated emitters, such as the one shown in $[32,55]$ (see Supplementary Figure S3 for SEM image). A microfabricated reservoir, created just beneath the capillary emitter, is first filled with ionic liquid and the liquid then reaches the tip of the emitter by capillary process. The extraction voltage is then applied. We have previously observed that these devices emit currents of up $\pm 2 \mu \mathrm{A}[35,55]$, in mixed iondroplet mode with emission half-angle of $40^{\circ}$ [32] at operating voltages between $\pm 700 \mathrm{~V}$ and $1000 \mathrm{~V}$. Retarding potential analysis (RPA) of these devices has previously shown that the kinetic energy of emission can have nearly $10 \%$ deficit from the applied emitter potential $V_{e m}[35,36]$. There can also be secondary peaks $[35,36]$ corresponding to ion defragmentation in which dimers can break into neutral molecules and a monomers during their flight and share the kinetic energy according to the ratio 
(a)

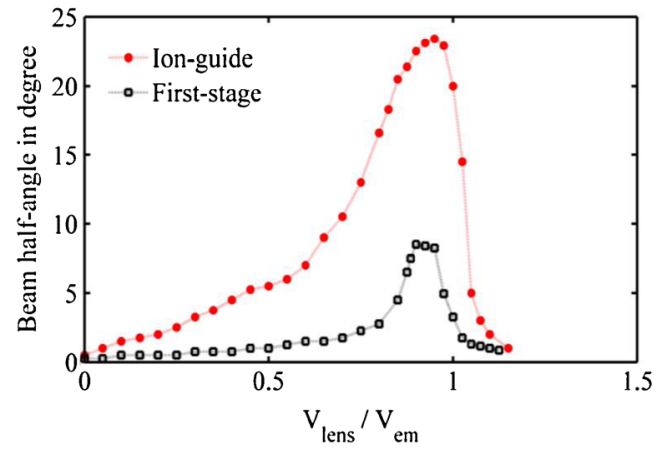

(b)

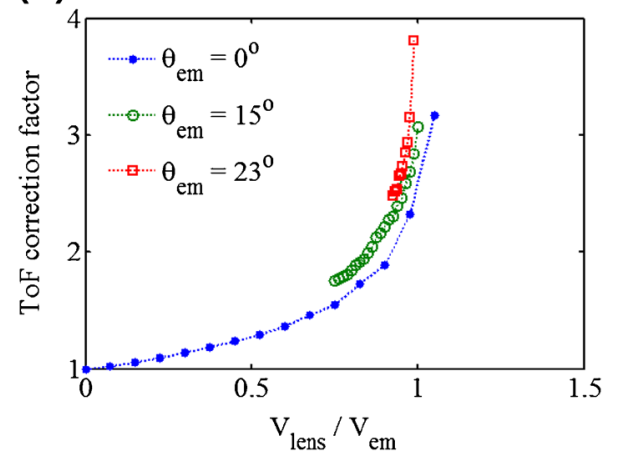

Figure 2. (a) Simulated plot of the maximum half-angle up to which the ion guide can collect $100 \%$ of the charges versus the ratio of the lens voltage over emitter voltage. The ion guide can collect all mono-energetic charges within $23^{\circ}$ halfangle, whereas for the first-stage it is only $9^{\circ}$. Also, nearly $30 \%$ energy spread can be collected within $15^{\circ}$ half-angle at a given lens voltage. (b) Simulated plot of the ToF correction factor versus $V_{\text {lens }} / V_{\text {em }}$ for charges emitted at different angles. The charges emitted at higher emission half-angles are delayed more than the ones closer to the axis, leading to slight spread in the ToF traces

of their masses. We, therefore, expect the fraction of charge collection to be lower for these capillary emitters than that for the needle emitters.

In Figure 6a, the fraction of current collected for different negative emitter voltages (for positive emission polarities, see Supplementary Figure S4) is shown for a capillary emitter with $10 \mu \mathrm{m}$ inner diameter with $150 \mu \mathrm{m}$ diameter extractor. Simulations have also been performed assuming a beam-shape similar to that reported in [32] and equal (a)

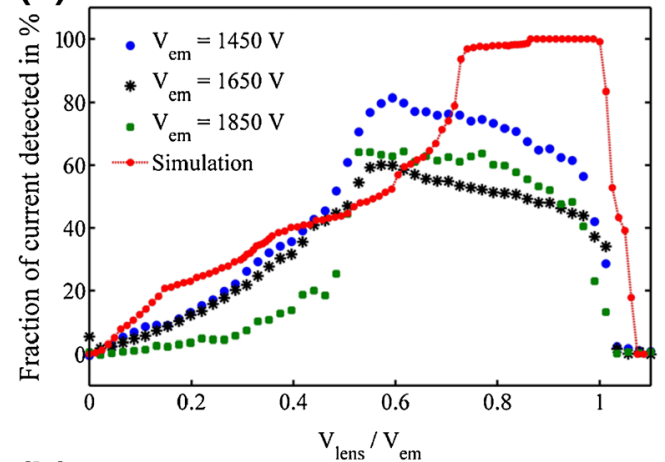

(b)

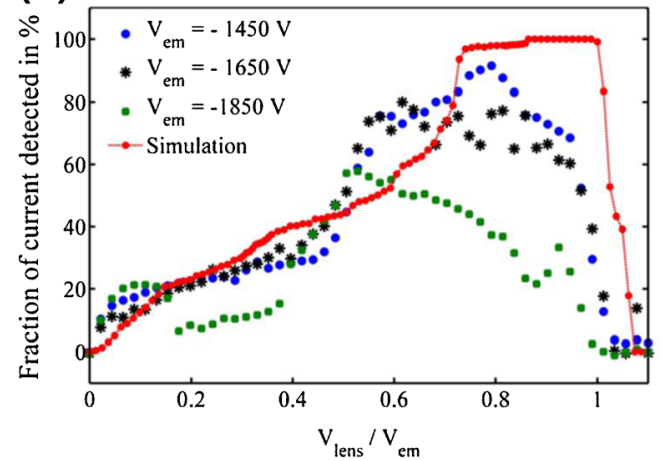

Figure 4. Plot of measured fraction of current collected from the tungsten needle electrospray emitter versus $V_{\text {lens }} / V_{\text {em }}$ for different positive (a), and negative (b) emitter voltages $V_{e m}$. Up to $80 \%$ of the emitted beam is collected at the end of the $65-\mathrm{cm}$ long flight tube. The simulation values have also been plotted for comparison. The simulations were performed assuming mono-energetic emission with kinetic energy = $q V_{e m}$ and the beam-shape reported in [47]

fraction of charges at two different kinetic energies given by Equations 4 and 5:

$$
\begin{aligned}
& (K . E .)_{1}=0.9 \times q V_{e m} \\
& (K . E .)_{2}=\frac{m_{\text {monomer }}}{m_{\text {dimer }}}(K . E .)_{1}
\end{aligned}
$$

where $m_{x}$ denotes the mass of the ion $x$. The simulated fraction values based on these assumptions are plotted in the same figures for comparison. It is found from the experi-

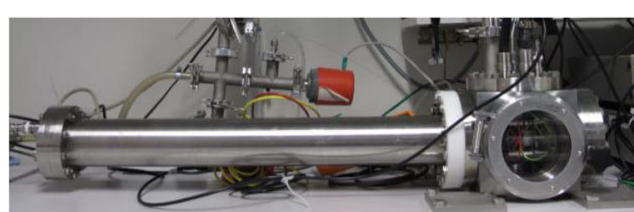

(a)

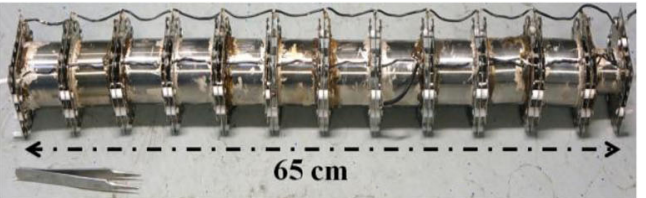

(b)

Figure 3. (a) Image of the ToF measurement setup, and (b) image of the 65-cm long ion guide. The ion guide slides inside the ToF tube. The emitter is assembled on the right side of the setup inside the cubic vacuum chamber and the Faraday cup is placed on the left end of the tube 
(a)

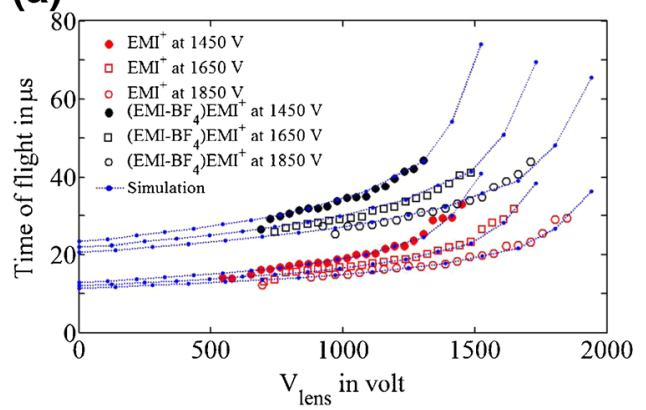

(b)

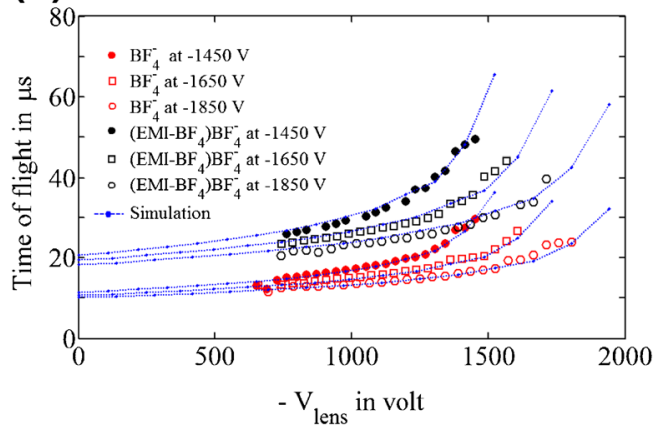

Figure 5. Plot of measured flight times of monomers and dimers from tungsten needle electrospray emitters versus lens voltages at three emitter voltages in both positive (a) and negative (b) polarity of emission, showing the increase in flight time with lens voltage. The simulated values are also shown in the same figure for comparison. The change of the ToF with $V_{\text {lens }}$ for the different species very closely follows the simulation results

ments that the collected current reaches maximum value of nearly $20 \%-30 \%$ at values of $V_{\text {lens }}$ corresponding to the two kinetic energies, very close to the simulated values shown in the figure. However, the experimental range with high collection fraction is broader than that obtained from simulations of a beam equally proportioned between energies $(K . E .)_{1}$ and $(K . E .)_{2}$. The simulations were performed assuming only two discrete energies and further kinetic energy spreading had not been taken into account for simplicity. Also, the emission from capillary emitters has been previously reported to comprise of significant proportion of droplets $[35,39,46]$ with kinetic energies distributed over a wide range. These droplets are collected for different lens voltages leading to the spread in the experimentally observed collection range. For the case of tungsten needles, the lack of a distinct low energy peak suggests that defragmentation was not as significant as in case of the capillary emitters. Furthermore, the assumptions of purely ionic and mono-energetic emission are consistent with the observations of low current collection at low $V_{\text {lens }}$.

ToF measurements were performed on the capillary emitters for different $V_{e m}$ with $V_{\text {lens }}=0.7 \times V_{e m}$ to ensure high fraction of charge collection. This leads to a ToF correction factor of 1.5. In Figure $6 \mathrm{~b}$, the ToF of the $\mathrm{BF}_{4}^{-}$
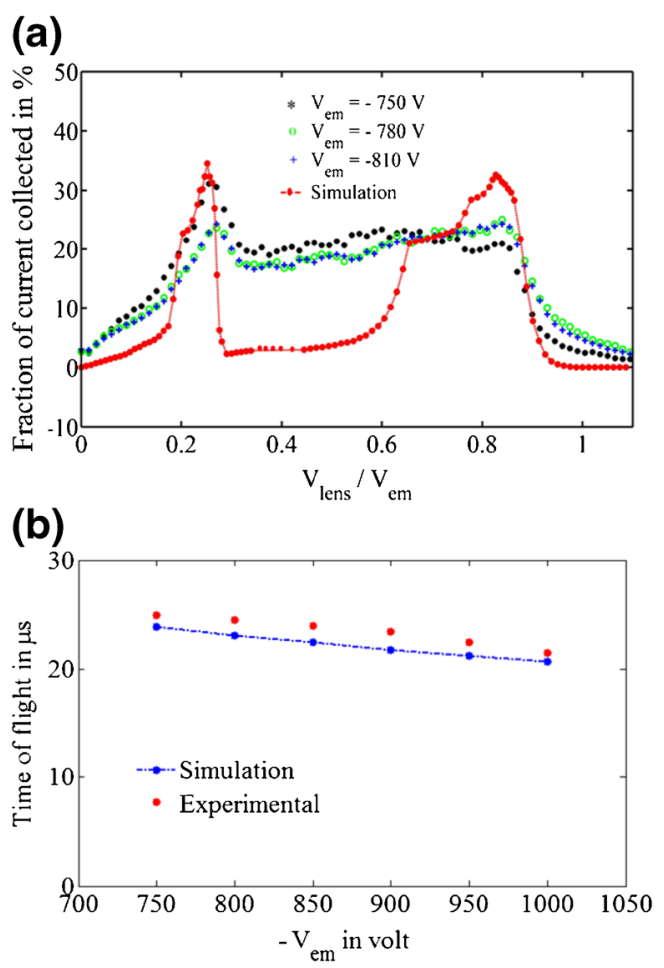

Figure 6. (a) Plot of the fraction of current detected from the microfabricated electrospray emitters versus $V_{\text {lens }} / V_{e m}$ in negative polarity. The simulation results assuming a beamshape as reported in [32] and two kinetic energies given by (4) and (5) with equal intensities are also shown in the same figures for comparison. (b) Plot of flight time of $\mathrm{BF}_{4}^{-}$ions versus $V_{e m}$ with $V_{\text {lens }}$ set to $0.7 \times V_{e m}$. The simulated plot of ToF of the same based on the ToF correction factor $=1.5$ and kinetic energy $=0.9 \times q V_{e m}$ is also shown in the same figure for comparison

ions is plotted against $V_{e m}$ for $V_{\text {lens }}=0.7 \times V_{e m}$. The simulated ToF values, based on ToF correction factor $=1.5$ and kinetic energy $=(\text { K.E. })_{1}=0.9 \times q V_{e m}$ for this case are also shown in the same figure. The results match within $4.5 \%$. The difference may be due to uncertainty of measurement from the spreading ToF traces and energy spread of the emitted species.

\section{Conclusions}

In this paper, we report the development of a time-of-flight mass spectrometer system that incorporates an ion guide covering the full length of the flight tube. The ion guide allows collecting at the Faraday cup charge from electrospray sources that emit with large beam half-angles and with wide energy spread. Our simulation shows that the ion guide is capable of collecting all mono-energetic charges emitted within a $23^{\circ}$ half angle about its axis and can collect all charges with $30 \%$ energy spread even with a nearly $15^{\circ}$ half-angle. The ion guide can compensate for under-focused or over-focused charges, which are otherwise not collected using single-lens systems. 
We first characterized the fraction of charge collection of the electrostatic ion guide with nearly mono-energetic ion beams from the ionic liquid EMI-BF $\mathrm{B}_{4}$ emitted from externally wetted tungsten needles and observed that $80 \%$ of the emitted charges can be detected for ToF measurements. The system was then used to characterize the spray behavior of microfabricated capillary emitters spraying droplets and ions from the ionic liquid and nearly $30 \%$ of the charges could be collected despite the large emission half-angle of over $40^{\circ}$. This allows characterizing the spray behavior over a large emission divergence of the emitted charges and is, thus, potentially capable of providing spray information from a large fraction of the emission. We note that this benefit must be traded against a reduction in $q / m$ sensitivity due to flight time variations by up to $20 \%$ over beam angle.

ToF measurements have been performed on pure ion beams as well as on mixed-mode electrospray (ions/droplet emission) and in both cases the measured ToF matched the predicted dependence on the focusing voltage very closely, showing the ion guide allows for accurate ToF timing acquisition, while collecting many times more current than could be possible with an Einzel lens. This means (1) the gain and speed requirements of the trans-impedance amplifier can be greatly relaxed, (2) a smaller Faraday cup and, hence, much smaller diameter flight tube can be used, and (3) a wider part of the beam can be collected, allowing a more complete characterization of the emitted species (not just the center of the beam).

Since the ion guide covers the full length of the flight tube, the collected charge is expected to be independent of the length of the flight tube and can be applied to many different ToF measurement configurations. The up to three times increase in flight time obtained by increasing the lens voltage can be a means of increasing the detectability without increasing the actual length of the flight tube.

\section{Acknowledgments}

The authors acknowledge Dr. C. N. Ryan from Queen Mary University, London, for important discussions. This work was partially supported by the Swiss National Science Foundation under grant 200021_146365, the ESA NPI programme, and the FP7 MicroThrust project, grant agreement number 263035, funded by the EC Seventh Framework Programme theme FP7-SPACE-2010.

\section{References}

1. Cotter, R.J.: Time-of-flight Mass Spectrometry: Instrumentation and Applications in Biological Research, 1st ed., ACS Professional Reference Books: Washington DC (1997)

2. Cameron, A.E., Eggers Jr., D.F.: An ion elocitron. Rev. Sci. Instrum. 19, 605-607 (1948)

3. Wiley, W.C., McLaren, I.H.: Time of flight mass spectrometer with improved resolution. Rev. Sci. Instrum. 26, 1150-1157 (1955)

4. Karas, M., Hillencamp, F.: Laser desorption ionization of proteins with molecular masses exceeding 10000 Daltons. Analytical Chemistry 60, 2291-2301 (1988)
5. Tanaka, K., Waki, H., Ido, Y., Akita, S., Yoshida, Y., Yoshida, T., Mastuo, T.: Protein and polymer analyses up to $m / z 100,000$ by laser ionization time-of-flight mass spectrometry. Rapid Commun. Mass Spectrom. 2, 151-153 (1988)

6. Cornish, T.J., Cotter, R.J.: Tandem time-of-flight mass spectrometer. Anal. Chem. 65, 1043-1047 (1993)

7. Fenn, J.B., Mann, M., Meng, C.K., Wong, S.F., Whitehouse, C.M.: Electrospray ionization for mass spectrometry of large biomolecules. Science 246, 64-71 (1989)

8. Fenn, J.B.: Electrospray ionization mass spectrometry: how it all began. J. Biomed. Tech. 13, 101-118 (2002)

9. Ho, C.S., Lam, C.W.K., Chan, M.H.M., Cheung, R.C.K., Law, L.K., Lit, L.C.W., Ng, K.F., Suen, M.W.M., Tai, H.L.: Electrospray ionization mass spectrometry: principles and clinical applications. Clin. Biochem. Rev. 24, 3-12 (2003)

10. Vestal, M.L.: The future of biological mass spectrometry. J. Am. Soc. Mass Spectrom. 22, 953-959 (2011)

11. Lozano, P.C.: Energy properties of an EMI-Im ionic liquid ion source. J. Phys. D Appl. Phys. 39, 126-134 (2006)

12. Lozano, P.: Studies on the ion-droplet mixed regime in colloid thrusters. PhD Dissertation, Department of Aeronautics and Astronautics, MIT, USA (2003)

13. Bamberger, C., Renz, U., Bamberger, A.: Digital imaging mass spectrometry. J. Am. Soc. Mass Spectrom. 22, 1079-1087 (2011)

14. Ebata, S., Ishihara, M., Kumondai, K., Mibuka, R., Uchino, K., Yurimoto, H.: Development of an ultra-high performance multi-turn TOF-SIMS/SNMS system MULTUM-SIMS/SNMS. J. Am. Soc. Mass Spectrom. 24, 222-229 (2013)

15. May, J.C., Russell, D.H.: A mass-selective variable-temperature drift tube ion mobility-mass spectrometer for temperature dependent ion mobility studies. J. Am. Soc. Mass Spectrom. 22, 1134-1145 (2011)

16. Contino, N.C., Pierson, E.E., Keifer, D.Z., Jarrold, M.F.: Charge detection mass spectrometry with resolved charge states. J. Am. Soc. Mass Spectrom. 24, 101-108 (2013)

17. Guilhaus, M.: Principles and Instrumentation in time-of-flight mass spectrometry. J. Mass Spectrom. 30, 1519-1532 (1995)

18. Mühlberger, F., Saraji-Bozorgzad, M., Gonin, M., Fuhrer, K., Zimmermann, R.: Compact ultrafast orthogonal acceleration time-of-flight mass spectrometer for on-line gas analysis by electron impact ionization and soft single photon ionization using an electron beam pumped rare gas excimer lamp as VUV-light source. Anal. Chem. 79, 8118-8124 (2007)

19. Kitahara, Y., Takahashi, S., Kuramoto, N., Sala, M., Tsugoshi, T., Sablier, M., Fujii, T.: Ion attachment mass spectrometry combined with infrared image furnace for thermal analysis: evolved gas analysis studies. Anal. Chem. 81, 3155-3158 (2009)

20. Basile, F., Zhang, S., Kandar, S.K., Lu, L.: Mass spectrometry characterization of the thermal decomposition/digestion (TDD) at cysteine in peptides and proteins in the condensed phase. J. Am. Society Mass Spectrom. 22, 1926-1940 (2011)

21. van Wuijckhuijse, A. L, van Baar, B. L. M.: Recent advances in realtime mass spectrometry detection of bacteria. In: Zourob, M., Elwary S., Turner, A. (eds.) Principles of Bacterial Detection: Biosensors, Recognition Receptors and Microsystems, pp. 929-954. Springer, New York (2008)

22. Arnold, R.J., Karty, J.A., Ellington, A.D., Reilly, J.P.: Monitoring the growth of a bacteria culture by MALDI-MS of whole cells. Anal. Chem. 71, 1990-1996 (1999)

23. Wilkes, J.G., Rafli, F., Sutherland, J.B., Rushing, L.G., Buzatu, D.A.: Pyrolysis mass spectrometry for distinguishing potential hoax materials from bioterror agents. Rapid Commun. Mass Spectrom. 20, 2383-2386 (2006)

24. Spraggins, J.M., Caprioli, R.M.: High-speed MALDI-TOF imaging mass spectrometry: rapid ion image acquisition and considerations for next generation instrumentation. J. Am. Soc. Mass Spectrom. 22, 10221031 (2011)

25. Romero-Sanz, I., Bocanegra, R., de la Moraa, J.F., Gamero-Castano, M.: Source of heavy molecular ions based on Taylor cones of ionic liquids operating in the pure ion evaporation regime. J. Appl. Phys. 94, 3599-3605 (2003)

26. Marcuccio, S., Genovese, A., Andrenucci, M.: Experimental performance of field emission microthrusters. J. Propulsion Power 14, 774781 (1998)

27. Zorzos, A.N., Lozano, P.C.: The use of ionic liquid ion sources in focused ion beam applications. J. Vacuum Sci. Technol. B 26, 20972102 (2008) 
28. Fedkiw, T.P., Lozano, P.C.: Development and characterization of an iodine field emission ion source for focused ion beam applications. J. Vacuum Sci. Technol. B 27, 2648-2653 (2009)

29. Lozano, P., Martínez-Sánchez, M.: Ionic liquid ion sources: suppression of electrochemical reactions using voltage alternation. J. Colloid Interface Sci. 280, 149-154 (2004)

30. Courtney, D.G.: Ionic liquid ion source emitter arrays fabricated on bulk porous substrates for spacecraft propulsion. PhD Dissertation, Department of Aeronautics and Astronautics, MIT, USA (2011)

31. Terhune, K.J., King, L.B., He, K., Cumings, J.: In situ visualization of ionic liquid electrospray emission using transmission electron microscopy. Proceedings of the 49th AIAA/ASME/SAE/ASEE Joint Propulsion Conference. San Jose, CA, July 14-17, p. 146 (2013)

32. Chakraborty, S., Ataman, C., Dandavino, S., Shea, H.: Microfabrication of an electrospray thruster for small spacecraft. Proceedings of POWERMEMS 2012. Atlanta, GA, December 2-5, p. 073 (2012)

33. Miller, S. W., Prince, B. D.: Capillary extraction of the ionic liquid $[\mathrm{Bmim}][\mathrm{DCA}]$ for variable flow rate operations. Proceedings of the 48th AIAA/ASME/SAE/ASEE Joint Propulsion Conference and Exhibit Atlanta, GA, July 30-August 1 (2012)

34. Gamero, C.M.: The structure of electrospray beams in vacuum. J Fluid Mech 604, 339-368 (2008)

35. Krpoun, R.: Micromachined electrospray thrusters for spacecraft propulsion. PhD Dissertation, EPFL, Switzerland (2009)

36. Krpoun, R., Shea, H.R.: Integrated out-of-plane nano-electrospray thruster arrays for spacecraft propulsion. J. Micromech. Microeng. 19, 045019 (2009)

37. Andersson, G.: Angle-resolved ion scattering spectroscopy at surfaces of pure liquids: topography and orientation of molecules. Phys. Chem. Chem. Phys. 7, 2942-2947 (2005)

38. Romero-Sanz, I., de la Mora, J.F.: Energy distribution and spatial structure of electrosprays of ionic liquids in vacuo. J. Appl. Phys. 4, 2123-2129 (2004)

39. Ryan, C., Daykin-Iliopoulos, A., Stark, J.A., Salaverri A., Vargas, E., Rangsten, P., Dandavino, S., Ataman, C., Chakraborty, S., Courtney, D., Shea, H.: Experimental progress towards the MicroThrust MEMS electrospray electric propulsion system. Proceedings of the 33rd International Electric Propulsion Conference, Washington DC, Oct. 6-10, p. 146 (2013)

40. Sise, O., Ulu, M., Dogan, M.: Multi-element cylindrical electrostatic lens systems for focusing and controlling charged particles. Nuclear Instrum. Methods Phys. Res. A: Accelerators, Spectrometers, Detectors, and Associated Equipment. 554, 114-131 (2005)

41. Riddle, G.H.N.: Electrostatic Einzel lenses with reduced spherical aberration for use in field emission guns. J. Vacuum Sci. Technol. 15, 857-860 (1978)
42. Imhof, R.E., Read, F.H.: A three-aperture electron optical lens for producing an image of variable energy but fixed position. J. Sci. Instrum. 1, 859-860 (1968)

43. Rohrbacher, A., Continett, R.E.: Multiple-ion-beam time-of-flight mass spectrometer. Rev. Sci. Instrum. 72, 3386-3389 (2001)

44. Gillig, K.J., Ruotolo, B.T., Stone, E.G., Russell, D.H.: An electrostatic focusing ion guide for ion mobility-mass spectrometry. Int. J. Mass Spectrom. 239, 43-49 (2004)

45. Blasé, R.C., Silveira, J.A., Gillig, K.J., Gamage, C.M., Russell, D.H.: Increased ion transmission in IMS: a high resolution, periodic-focusing DC ion guide ion mobility spectrometer. Int. J. Mass Spectrom. 301, 166-173 (2011)

46. Dandavino, S., Ataman, C., Chakraborty, S., Shea, H., Ryan, C., Stark J.: Design and fabrication of the thruster heads for the MicroThrust MEMS electrospray propulsion system. Proceedings of the 33rd International Electric Propulsion Conference. Washington DC, Oct. 610, p. 127 (2013)

47. Lozano, P., Martínez-Sánchez, M.: Ionic liquid ion sources: characterization of externally wetted emitters. J. Colloid Interface Sci. 282, 415421 (2005)

48. Variable gain High Speed Current Amplifier DHPCA-100. Available at: http://www.femto.de/en/products/current-amplifiers/variable-gain-up-to200-mhz-dhpca.html. Accessed 28 Nov 2013

49. Part Types Gallery. Available at: http://www.kimballphysics.com/evparts/technology/product-overview. Accessed 28 Nov 2013

50. Castro, S., de la Mora, J.F.: Effect of tip curvature on ionic liquid emission from Taylor cones of ionic liquids from externally wetted tungsten tips. J. Appl. Phys. 105, 034903 (2009)

51. Regulated products. Available at: http://www.emcohighvoltage.com/ regulated-power-supply.php. Accessed 28 Nov 2013

52. Precision, Wide Bandwidth 3-Port Isolation Amplifier: AD210. Available at: http://www.analog.com/static/imported-files/data_sheets/ AD210.pdf. Accessed 28 Nov 2013

53. PVX-4140 Datasheet. Available at: http://www.ixyscolorado.com/ index2.php?page=shop.product_details\&flypage=flypage.tpl\&product_id=26\& category id $=25 \&$ option $=$ com virtuemart\&Itemid $=29$. Accessed 28 Nov 2013

54. Larriba, C., Castro, S., de la Mora, J.F., Lozano, P.: Mono-energetic source of kilodalton ions from Taylor cones of ionic liquids. J. Appl. Phys. 101, 084303 (2007)

55. Ataman, C., Dandavino, S., Shea H.: Wafer-level integrated electrospray emitters for a pumpless microthruster system operating on high efficiency ion-mode. Proceedings of MEMS 2012, Paris, France, 1293-1296 (2012) 